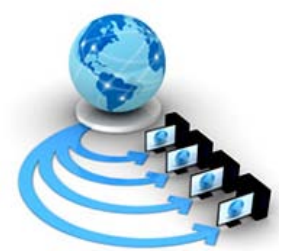

Volume 9, No. 1, January-February 2018

International Journal of Advanced Research in Computer Science

RESEARCH PAPER

\author{
Available Online at www.ijarcs.info
}

\title{
A SHORT REVIEW ON IMAGE RESTORATION OF NOISY AND BLURRED IMAGES AND COMPARISON OF STATE OF ART ALGORITHMS
}

\author{
Rachana Dhannawat \\ Assitant Professor CSE department \\ Usha Mittal Institute of Technology, SNDT university \\ Mumbai, India
}

\author{
Dr. Archana B. Patankar \\ Associate Professor, CSE depatment \\ Thadomal Shahani Engineering College \\ Mumbai, India
}

\begin{abstract}
This paper analyzes most of restoration method frequently used and gives comparison of seven recent algorithms in terms of working and applicability It compares algorithms like KSVD, BM3D, CSR, KLLD, SVD based, LPGPCA, NCSR and many other spatial domain, transform domain and dictionary based methods, iterative methods for image restoration of noisy and blurred images. It gives comparative survey of all restoration techniques which will be useful to researchers for further development in the field.
\end{abstract}

Keywords:KSVD; KLLD; NCSR; BM3D; CSR; SVD based; LPGPCA; Dictionary learning; Transform domain; Spatial domain; ISTA; FISTA; TWIST.

\section{INTRODUCTION}

Image Restoration is process of regaining the original image from its degraded version [1]. It includes image denoising [28], deblurring, superresolution [1]. It is an ill posed problem and many researchers tried to solve it in various ways. These can be broadly classified and discussed in rest of the paper.

\section{Classification OF IMAgE RESTORATION TECHNIQUES}

The vast work done in the area can be classified in following heads.

\section{A. Local and Non local filters}

Many local filters such as Gaussian filter, median filter [3], inverse filter Wiener filter[2], Least Mean Squares filter, bilateral filter [6][7], joint bilateral filter, Lee filter, etc. are used for noise reduction but as compared to them non local filter always gives better results. The nonlocal filters make use of the self-similarity of natural images in a nonlocal manner. The basic NLMwas developed in[4], later many improvements to algorithm are proposed [5].

\section{B. Transform Domain Techniques:}

These include wavelets, BM3D, LPGPCA, and LPGSVD. Noise is spread out uniformly in wavelet domain while signal gets concentrated in few significant components. This is called as sparsity property. Using this concept different wavelet transforms can be used for image restoration. Discrete Wavelet Transform and thresholding is used to get denoised image in [8] and DWT is applied on restored image to get better quality image in [9]. Wavelet based EM algorithm for multispectral images is proposed in [10] [11].Noise is first reduced by adaptively shrinking wavelet coefficients using alpha map [13] and entropy, and then a new directional transform using combined wavelet functions and an adaptive Gaussian low-pass filter is composed in [12]. In [15] researchers have compared 17 methods for Image Denoising using wavelet transforms where various types of noise, transforms and thresholding are compared. It indicates vast work done by researchers in the area. With

wavelets usually five thresholding methods are used to reduce the noises that are hard thresholding, soft thresholding, VisuShrink, SureShrink, BayesShrink. [8][12][14]. Block Matching and 3-D Filtering (BM3D) based on variation of $\mathrm{K}$ nearest neighbor clustering and 2 stage simplification of EM based estimation of signal variance was developed [16]. Improvements [17] [18] to it are also proposed and used for image restoration. LPGPCA includes 3 steps local pixel grouping, PCA transform and inverse PCA transform [19]. LPGSVD [20] is similar technique to LPGPCA. It also has 3 steps local pixel grouping, SVD computation followed by aggregation.

\section{Vector Quantization}

Blind image restoration algorithm based on Vector Quantization was proposed by Aggelos K. Katsaggelos et.al. [21] [22] [23].

\section{Regularization}

Regularization can be iterative or direct. Regularization has a general form as

$$
\operatorname{Min} \mid\|\mathrm{y}-\mathrm{Hx}\|_{2}^{2}+\lambda\|\mathrm{x}\|_{0}
$$

Where $\lambda$ is regularization parameter.

Many variations to this basic equation are developed and known as L1 norm, Lp norm, Tikhonov, L1/L12, Total Variation [24], Mumford Shah [27] [29] , Sparsity [25] [30] [33] etc. Using Sparsity property of image many recent methods of Image Restoration [28] are developed such as KSVD [31] [32], Learned Simultaneous Sparse Coding (LSSC) [34], and Clustering-based Sparse Representation 
(CSR) [35], Non locally Centralized Sparse Representation(NCSR) [1] [36], Clustering-based Denoising with locally learned dictionaries (KLLD) [38] [37]. These are explained below in short.

\section{E. KLLD [38]}

This algorithm applies K-means clustering to images. On these clusters PCA transform is applied and then dictionary is formed. It uses steering Kernel Regression for weight calculation [38].

\section{F. CSR [35]}

The steps in algorithm are K-means clustering, followed by PCA then shrinkage algorithm. In last step it uses L1 optimization as regularization process. It does not need any initial dictionary.

\section{G. Nonlocally Centralized Sparse Representation (NCSR) [1] [36]}

This algorithm is combination of all recent image restoration techniques. It begins with L1 regularization with sparse representation. It improves sparse representation by proposing non locally centralized sparse representation in which image is divided into overlapping patches. It also uses iterative shrinkage algorithm for solving L1 regularized least square problem. It used uses K-means clustering and PCA to form dictionary. To calculate estimation of sparse codes it uses weighted average formula similar to non local means algorithm. It also used maximum a posterior estimation (MAP) for evaluating regularization parameter $\lambda$. This algorithm works for denoising, deblurring and super resolution.

\section{H. Direct Regularization}

Truncated SVD [26]: SVD [53] [54] solution is given by $\mathrm{A}=$ $\mathrm{U} \sum \mathrm{V}$. If we approximate the SVD solution by considering some rank k matrix is known as truncated SVD solution.

\section{Iterative}

In iterative algorithms, during the iterations the blurred version of the current restoration result is compared to the recorded image. The difference between the two is scaled and added to the current restoration result to give the next restoration result. Various iterative algorithms are Van Cittert Algorithm [39] [40], Landweber Algorithm [39] [41] [42] [43], Poisson Map Algorithm [39], Richardson-Lucy Algorithm [39], Iterative Shrinkage Thresholding algorithm (ISTA) [44], Fast Iterative Shrinkage Thresholding algorithm (FISTA) [45] [46], Two step Iterative Shrinkage Thresholding algorithm (TwIST)[47]. ISTA [44] implements Landweber algorithm followed by soft thresholding [8] for restoration. FISTA [45] [46] is same as ISTA except that iterative shrinkage step considers two previous points for calculation instead of one point. TwIST [47] combines advantages of two methods ISTA and Iterative Re-weighted Shrinkage (IRS) [55] [56] algorithm. It gives good denoising performance as IST algorithm and speed as IRS algorithm.

\section{J. Expectation Minimization (EM) [48] algorithm} The Expectation Minimization (EM) [48] algorithm is a general procedure for finding maximum likelihood parameter estimates. It consists of two steps Expectation step and Maximization step. By alternating the E-step and the M-step, convergence to an optimum of the likelihood function is achieved.

\section{K. Bayesian Estimator:}

Bayesian estimators [49] [50] includes estimators such as Maximum a posterior estimation (MAP), Maximum Likelihood Estimation (ML), minimum mean square error (MMSE), minimum mean absolute value of error (MAVE), etc.

\section{Fusion:}

Fusion of two or more restored images can be done to improve quality of restored image. Wavelet based fusion of two restored images by two different algorithms like Lucy Richardson, Wiener are used in [51] [52].

Table I compares state of art algorithms and illustrates that all of them use as basic steps pixel grouping or clustering followed by PCA or SVD as decomposition method.

Table I. Summary of basic steps in all state of art algorithms

\begin{tabular}{|c|l|}
\hline Technique & \multicolumn{1}{c|}{ Steps of Algorithm } \\
\hline KSVD [31] & Orthogonal Matching pursuit $\rightarrow$ SVD \\
\hline KLLD [38] & Clustering $\rightarrow$ PCA $\rightarrow$ Form Dictionary \\
\hline NCSR [1] & L1 Optimization $\rightarrow$ K means Clustering $\rightarrow$ PCA $\rightarrow$ Form Dictionary $\rightarrow$ Shrinkage Algorithm \\
\hline LPGPCA [19] & Local Pixel Grouping $\rightarrow$ PCA $\rightarrow$ Inverse PCA \\
\hline SVD based [20] & Local Pixel Grouping $\rightarrow$ SVD $\rightarrow$ Aggregation \\
\hline BM3D [16] & 3D transform $\rightarrow$ Thresholding (Advanced version uses PCA) \\
\hline CSR [35] & K means Clustering $\rightarrow$ PCA $\rightarrow$ Shrinkage Algorithm $\rightarrow$ L1 Optimization \\
\hline
\end{tabular}




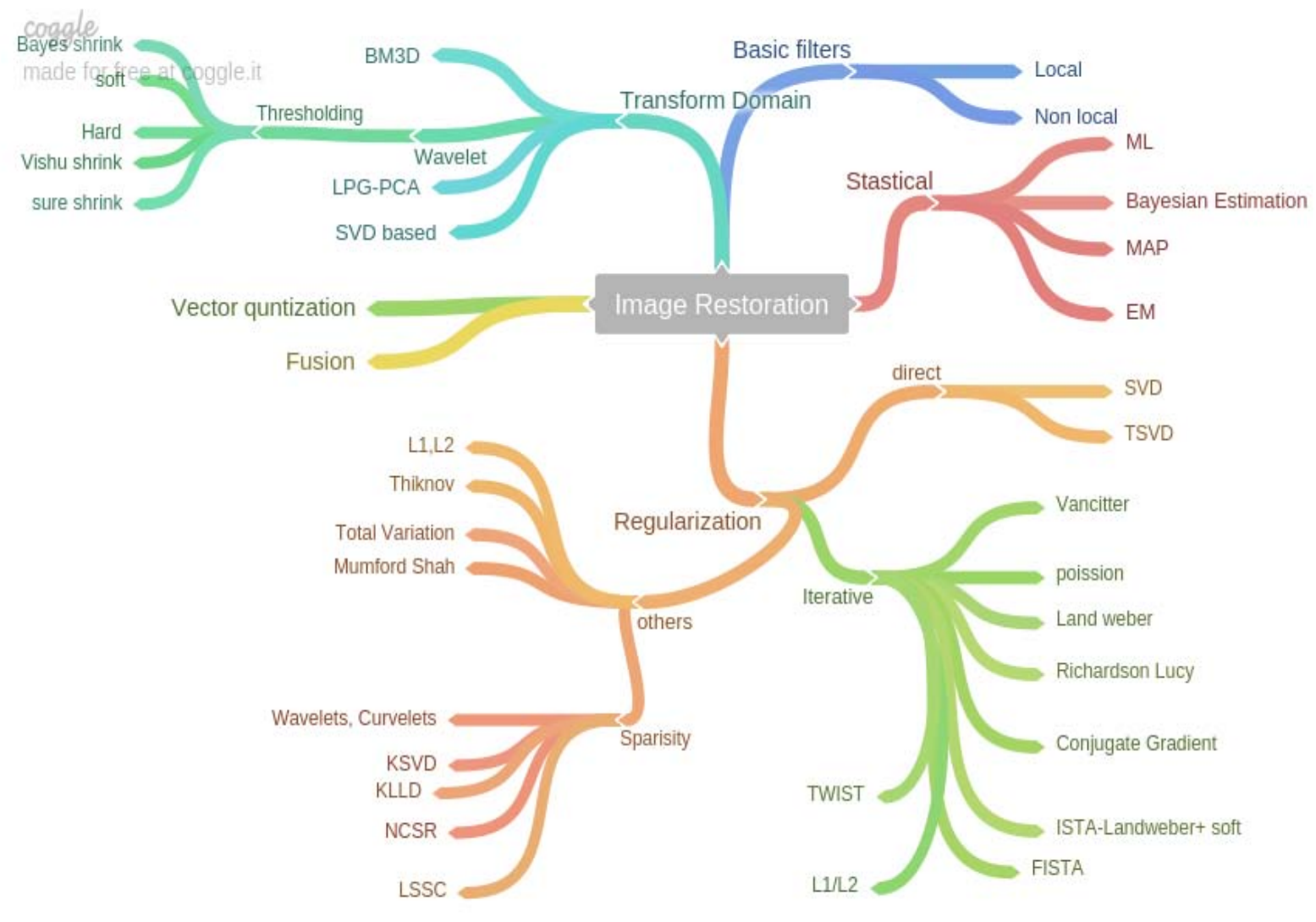

Figure1: Complete mind map of Image Restoration techniques.

Figure1 gives complete mind map of Image Restoration techniques discussed in the paper.

\section{CONCLUSION}

This paper compared many restoration techniques and analyzed based on working and applicability. Out ofall the algorithms discussedmaximum basic filters and above compared algorithms in table 1 work for image denoising. For image deblurring inverse filter and all iterative techniques are used dominantly in literature but it can also be done by direct methods. NCSR gives results for denoising, deblurring and super resolution.

\section{REFERENCES}

[1] Weisheng Dong, Lei Zhang,Guangming Shi, Xin Li, "Nonlocally Centralized Sparse Representation for Image Restoration”, IEEE Transactions on Image Processing, Vol. 22, No. 4, April 2013, pp-1620-1630.

[2] Jae-Chern Yoo, Chang Wook Ahn,” Image restoration by blind-Wiener filter,” IET Image Processing, Vol. 8, Iss. 12, 2014, pp. 815-823.

[3] Rakesh M.R, Ajeya B, Mohan A.R., "Hybrid Median filter For Impulse Noise Removal of an Image in Image Restoration”, International Journal of Advanced Research in Electrical, Electronics and Instrumentation Engineering, Vol. 2, No. 10, October 2013, pp-5117-5124.

[4] Antoni Buades, Bartomeu Coll, Jean-Michel Morel,” A nonlocal algorithm for image denoising,” IEEE International Conference on Computer Vision and Pattern Recognition, Vol. 2, 2005, pp- 60-65.

[5] Lu Lu, Weiqi Jin, Xia Wang,”Non Local Means Image Denoising with a Soft Threshold”, IEEE Signal Processing Letters, Vol. 22, No.7, July 2015, pp-833-837.
[6] C. Tomasi__ R. Manduchi,”Bilateral Filtering for Gray and Color Images”, IEEEInternational Conference on Computer Vision, 1998, pp-839-846.

[7] Ming Zhang and Bahadir K. Gunturk,” Multiresolution Bilateral Filtering for Image Denoising”, IEEE Transactions on Image Processing, Vol. 17, No. 12, December 2008, pp2324-2333.

[8] Rubeena Vohra, Akash Tayal, "Image Restoration Using Thresholding Techniques on Wavelet Coefficients “, International Journal of Computer Science (IJCSI), Vol. 8, No. 5, No 3, September 2011, pp-400-404.

[9] Archee Naz, Anjan Talukdar, Kandarpa Sarma, ”Digital Image Restoration using Discrete Wavelet Transform based Approach “,IRNet Transactions on Electrical and Electronics Engineering (ITEEE), Vol. 1, No. 2, 2012, pp-53-57.

[10] Mario A. T. Figueiredo, Robert D. Nowak, “An EM Algorithm for Wavelet-Based Image Restoration”, IEEE transactions on Image Processing, Vol. 12, No. 8, 2003, pp906-916.

[11] Arno Duijster, Paul Scheunders, and Steve De Backer,” Wavelet-Based EM Algorithm for Multispectral Image Restoration”, IEEE transactions on Geoscience and Remote Sensing, Vol. 47, No. 11, November 2009.

[12] Sangjin Kim, Wonseok Kang, Eunsung Lee, Joonki Paik, "Vaguelette-Wavelet Decomposition for Frequency Adaptive Image Restoration Using Directional Wavelet Bases”, IEEE Transactions on Consumer Electronics, Vol. 57, No. 1, February 2011, pp-218-223.

[13] Sangjin Kim, Sinyoung,Eunsung,Jeongho, Joonki Paik,” Real Time Bayer Domain Image Restoration for an Extended Depth of Field(EDoF) Camera", IEEE transactions on Consumer Electronics, Vol. 55, No. 2, November 2009, pp1756-1764. 
[14] Mantosh Biswas, Hari Om, “An Image Threshold Estimation Method ", Advances in Computer Science and its Applications (ACSA), Vol. 2, No. 3, 2013, pp-377-380.

[15] Pankaj Rakheja, Rekha Vig,” Image Denoising Using Various Wavelet Transforms: A Survey”, Indian Journal of Science and Technology, Vol. 9, No. 48, December 2016, page(s): 1- 7.

[16] Kostadin Dabov, Alessandro Foi, Vladimir Katkovnik, Karen Egiazarian, "Image Denoising by Sparse 3-D Transform-Domain Collaborative Filtering”, IEEE transactions on Image Processing, Vol. 16, No. 8, August 2007, pp-2080-2095.

[17] Longzhi Wang, Zhuo Meng, X. Steve Yao, Tiegen Liu, Ya Su, and Mingliang Qin," Adaptive Speckle Reduction in OCT Volume Data Based on Block-Matching and 3-D Filtering”, IEEE Photonics Technology Letters, Vol. 24, No. 20, October 2012, pp-1802-1804.

[18] Matteo Maggioni, Vladimir Katkovnik, Karen Egiazarian, Senior Member, IEEE, and Alessandro Foi,” Nonlocal Transform-Domain Filter for Volumetric Data Denoising and Reconstruction”, IEEE transactions on Image Processing, Vol. 22, No. 1, January 2013, pp-119-133.

[19] Lei Zhang, WeishengDong, DavidZhang, GuangmingShi,” Two-stage image denoising by principal component analysis with local pixel grouping", Elsevier journal on Pattern Recognition vol. 43, 2010, pp-1531-1549.

[20] Qiang Guo, Caiming Zhang, Yunfeng Zhang, and Hui Liu,” An Efficient SVD-Based Method for Image Denoising”, IEEE transactions on Circuits and Systems for Video Technology, Vol. pp, No. 99, March 2015, pp-1-13.

[21] Ryo Nakagaki,Aggelos K. Katsaggelos,” A VQ-Based Blind Image Restoration Algorithm”, IEEE transactions on Image Processing, Vol. 12, No. 9, September 2003, pp-1044-1053.

[22] Ryo Nakagaki,Aggelos K. Katsaggelos,”A VQ- Based Blur Identification Algorithm ", IEEEInternational Conference on acoustics, speech and signal processing, April 2003, pp-725728.

[23] Ryo Nakagaki,Aggelos K. Katsaggelos, “ A VQ-Based Image Restoration Algorithm”, IEEE International Conference on Image Processing, September 2002, pp-305308.

[24] Jose M. Bioucas-Dias, Mario A. T. Figueiredo, and Joao P. Oliveira," Total Variation-Based Image deconvolution: A Majorization-Minimization Approach”, IEEEInternational Conference on acoustics, speech and signal processing, France, 2006.

[25] Dilip Krishnan, Terence Tay, Rob Fergus,” Blind Deconvolution Using a Normalized Sparsity Measure”, IEEE International Conference onComputer Vision and Pattern Recognition, August 2011, pp-233-240.

[26] Ajit Rajwade, , Anand Rangarajan, Arunava Banerjee,” Image Denoising Using the Higher Order Singular Value Decomposition", ", IEEE transactions on Pattern Analysis and Machine Intelligence, Vol.35, No. 4, April 2013, pp849-862.

[27] Leah Bar, Nir Sochen, and Nahum Kiryati, "Semi-Blind Image Restoration Via Mumford-Shah Regularization”, IEEE Transactions on Image Processing, Vol. 15, no. 2, February 2006, pp-483-493.

[28] Wenyi Zhao and Art Pope,” Image Restoration Under Significant Additive Noise,” IEEE Signal Processing Letters, Vol. 14, No. 6, June 2007,pp 401-404.

[29] Miyoun Jung, Xavier Bresson, Tony F. Chan, and Luminita A. Vese," Nonlocal Mumford-Shah Regularizers for Color Image Restoration", IEEE Transactions on Image Processing, Vol. 20, No. 6, June 2011, pp-1583-1598.
[30] J. A. Tropp and S. J.Wright, "Computational methods for sparse solution of linear inverse problems," Proceedings of IEEE, vol. 98, no. 6, pp. 948-958, Jun. 2010.

[31] Michal Aharon, Michael Elad, and Alfred Bruckstein, "KSVD: An Algorithm for Designing Overcomplete Dictionaries for Sparse Representation”, IEEE transactions on Signal Processing, vol. 54, No. 11, November 2006, pp4311-4322.

[32] Michael Elad and Michal Aharon, "Image Denoising via Sparse and Redundant Representations over Learned Dictionaries”, IEEE transactions on Image Processing, Vol. 15, No. 12, December 2006, pp-3736-3745.

[33] Siddharth Saxena, Rajeev Kumar Singh,”A Novel Approach of Image Restoration Based on Segmentation and Fuzzy Clustering”,International Journal of Signal Processing, Image Processing and Pattern Recognition, Vol.7, No.4, 2014, pp-255-264.

[34] Weisheng Dong, Xin Li, Lei Zhang, and Guangming Shi, "Sparsity based image denoising via dictionary learning and structure clustering," IEEE International Conference onComputer Vision and Pattern Recognition CVPR, 2011, Vol. 2, pp- 457-464.

[35] J. Mairal, F. Bach, J. Ponce, G. Sapiro, and A. Zisserman, "Non-local sparse models for image restoration," IEEE 12th International Conference on Computer Vision, 2009, pp. 2272-2279.

[36] W. Dong, L. Zhang, G. Shi, and X. Wu, "Image deblurring and superresolution by adaptive sparse domain selection and adaptive regularization," transactions on Image Processing, vol. 20, no. 7, pp. 1838-1857, Jul. 2011.

[37] Wang, Chang-peng, and Jiang-she Zhang. "Image Denoising via Clustering-Based Sparse Representation over Wiener and Gaussian Filters", 2012 Spring Congress on Engineering and Technology, 2012, pp 1-4.

[38] Priyam Chatterjee, Peyman Milanfar,” Clustering-Based Denoising With Locally Learned Dictionaries”, IEEE transactions on Image Processing, Vol. 18, No. 7, July 2009, pp-1438-1451.

[39] Zohair Al-Ameen1, Ghazali Sulong, Md. Gapar Md. Johar,” A comprehensive study on Fast image deblurring techniques”, International Journal of Advanced Science and Technology, Vol.44, July 2012, pp. 1-11.

[40] Bennia and S. M. Riad, "Filtering Capabilities and Convergence of the Van-Cittert Deconvolution Technique", IEEE Transactions on Instrumentation and Measurement, vol. 41, No. 2, (1992), pp. 246-250.

[41] L. Lang and Y. Xu, "Adaptive Landweber method to deblur images”, IEEE Signal Processing Letters, vol. 10, no. 5, (2003), pp. 129-132.

[42] Swati Sharma, Shipra Sharma, Rajesh Mehra, "Image Restoration using Modified Lucy Richardson algorithm in presence of Gaussian and motion blur”, Research India publication Advance in Electronic and Electrical Engineering, Vol. 3, No.8, 2013, pp.509-523.

[43] Cedric Vonesch, Michael Unser," A Fast Multilevel Algorithm for Wavelet-Regularized Image Restoration”, IEEE transactions on Image Processing, Vol. 18, No. 3, March 2009, pp-2080-2095.

[44] Daubechies, M. Defriese, and C. DeMol, “An iterative thresholding algorithm for linear inverse problems with a sparsity constraint,” Commun. Pure Appl. Math., vol. 57, no. 11, pp. 1413-1457, 2004

[45] Amir Beck, Marc Teboulle,” A Fast Iterative Shrinkage Thresholding Algorithm With Application ToWaveletBased Image Deblurring”, IEEEInternational Conference on acoustics, speech and signal processing, April 2009, pp-693696. 
[46] Amir Beck, Marc Teboulle,”A Fast Iterative ShrinkageThresholding Algorithm for Linear Inverse Problems”,SIAM Journal on Imaging Sciences ,Society for Industrial and Applied Mathematics, 2009 Vol. 2, No. 1, pp. 183-202.

[47] J. M. Bioucas-Dias and M. A. T. Figueiredo, "A new TwIST: Two-step iterative shrinkage/thresholding algorithms for image restoration," IEEE transactions on Image Processing, vol. 16, no. 12, pp. 2992-3004, Dec. 2007.

[48] Chao Dong,Meihua Xie," A blind image restoration algorithm based on Nonlocal Means and EM algorithm”, IEEE International Conference on Audio, Language and Image Processing, 2012, pp- 485-489.

[49] Joao P. Oliveira, Mário A. T. Figueiredo, Jose M. Bioucas Dias," Parametric Blur Estimation for Blind Restoration of Natural Images: Linear Motion and Out-of-Focus", IEEE transactions on Image Processing, Vol. 23, No. 1, January 2014, pp-466-476.

[50] Chao Wang, Yong Yue, Feng Dong, Yubo Tao, Xiangyin Ma, Gordon Clapworthy, Hai Lin, and Xujiong Ye," Nonedge-Specific Adaptive Scheme for Highly Robust Blind Motion Deblurring of Natural Imagess”, IEEE transactions on Image Processing, Vol. 22, No. 3, March 2013, pp-884897.
[51] Arun Kumar Patel, Nitin Muchhal, R.N. Yadav, "Method for Image Restoration using Wavelet based Image Fusion",International Journal of Computer Applications (IJCA), Vol.39, No.13, February 2012, pp-18-23.

[52] Rama Singh, Neelesh Gupta, Meha Shrivastava, "Restoration of Noisy Blur Image using Wavelet based Image Fusion”,International Conference on Cloud, Big Data and Trust, 2013, pp-18-23.

[53] Yanmin He, Tao Gan, Wufan Chen, and Houjun Wang," Adaptive Denoising by Singular Value Decomposition”, IEEE Signal Processing Letters, Vol. 18, No.4, 2011, pp. 215-218.

[54] David S. Watkins. Fundamentals of Matrix Computation. New Jersey, USA, 2010, pp. 259-526.

[55] Chen Chen, Junzhou Huang, Lei He Hongsheng Li," Preconditioning for Accelerated Iteratively Reweighted Least Squares in Structured Sparsity Reconstruction”, IEEE International Conference onComputer Vision and Pattern Recognition, September 2014, pp- 2713-2720.

[56] Chen Chen, Junzhou Huang, Lei He Hongsheng Li,” Fast Iteratively Reweighted Least Squares Algorithm for Analysis-Based Sparsity Reconstruction”, IEEE transactions on Pattern Analysis and Machine Intelligence, April 2015, available at arXiv:1411.5057v3 